Itinéraires Itinéraires

Littérature, textes, cultures

\title{
De la littérature au jeu vidéo : le spectre de la narration interactive
}

From Literature to Video Game. The Spectrum of Interactive Narrative

\section{Martin Ringot}

\section{(2) OpenEdition}

1 Journals

Édition électronique

URL : http://journals.openedition.org/itineraires/3948

DOI : $10.4000 /$ itineraires.3948

ISSN : 2427-920X

Éditeur

Pléiade

\section{Référence électronique}

Martin Ringot, « De la littérature au jeu vidéo : le spectre de la narration interactive », Itinéraires [En ligne], 2017-3| 2018, mis en ligne le 15 juin 2018, consulté le 01 octobre 2020. URL : http:// journals.openedition.org/itineraires/3948 ; DOI : https://doi.org/10.4000/itineraires.3948

Ce document a été généré automatiquement le 1 octobre 2020.

\section{(c) (i) (9) $\Theta$}

Itinéraires est mis à disposition selon les termes de la licence Creative Commons Attribution - Pas d'Utilisation Commerciale - Pas de Modification 4.0 International. 


\title{
De la littérature au jeu vidéo : le spectre de la narration interactive
}

\author{
From Literature to Video Game. The Spectrum of Interactive Narrative
}

Martin Ringot

\section{Introduction}

Ainsi donc, le réseau des possibles peut se concentrer dans les quelques pages d'un récit de Borges, tout comme il peut servir d'armature à des romans de longue ou de très longue portée, chaque partie reproduisant alors la densité du modèle. Mais aujourd'hui, ajouterai-je, la règle qui veut qu'on « fasse court » se trouve confirmée par les longs romans eux-mêmes, structurellement voués à accumuler, à moduler, à combiner. (Calvino $2003: 101$ )

Dans la dernière des cinq conférences qu'il a données à Harvard en 1985 sur l'avenir de la littérature, Italo Calvino développe la notion de «multiplicité ». Il s'inscrit dans une lignée d'auteurs qui recherchent, à l'instar de Paul Valéry, une œuvre qui puisse enfermer une certaine totalité, qu'elle soit encyclopédique ou narrative. À l'instar de la littérature médiévale qui tendait, comme la Divine Comédie, à intégrer tout le savoir dans une forme systémique et compacte ${ }^{1}$, la littérature moderne tend à une multiplicité narrative, où chaque récit en contient un autre en puissance, suppose un récit parallèle ou contraire, où une situation initiale donne lieu à une multiplicité de développements divergents. C'est ainsi qu'il propose la notion d'hyper-roman, pour laquelle il donne l'exemple de Si par une nuit d'hiver un voyageur, ou encore de La vie mode d'emploi de Georges Perec.

2 Si Calvino pense à ces deux œuvres pour donner une idée de la direction que doit prendre la littérature au nouveau millénaire, c'est pour leur capacité à intégrer une 
multiplicité de récits, qu'ils soient simultanés ou alternatifs. S'interroger sur la littérature expérimentale revient à se demander quelle sera la prochaine grande évolution dans la manière dont un texte est créé. Il y a un siècle, cinéma et photographie ont poussé les écrivains à dire autrement la réalité, en brisant les formes poétiques et en mettant à mal la fiabilité de la représentation littéraire. Aujourd'hui, c'est le numérique et son fonctionnement procédural qui invitent les auteurs à repenser la création littéraire comme mise en place d'un système narratif. Qu'est-ce que cette avancée technologique a permis en termes d'expérience littéraire? Ajoutons que le numérique est venu aussi avec son lot de nouvelles expériences, notamment la possibilité pour une personne d'interagir avec un environnement généré à l'écran à l'aide d'un dispositif, et la littérature numérique ne peut être étudiée indépendamment de ces nouvelles pratiques qui proposent à leur tour un système narratif, lequel n'est pas étranger à cette multiplicité narrative que Calvino posait, dès 1985, comme l'une des pierres angulaires de la littérature du nouveau millénaire.

C'est pourquoi nous proposons une étude en deux temps. Tout d'abord, nous verrons comment le texte littéraire a connu dans la seconde moitié $\mathrm{du} \mathrm{xx}^{\mathrm{e}}$ siècle une nouvelle approche tant dans sa création que dans la conception que l'on se fait de l'objet-texte en soi. Cette modification de l'approche se confirmera par l'arrivée de la littérature dans l'environnement numérique, où des expériences de littérature combinatoire et interactive permettront le développement de l'hypertexte. Mais il n'a pas fallu attendre que la littérature s'empare de l'outil numérique pour voir apparaître des hypertextes. En effet - et ce sera le propos de notre second temps - le jeu vidéo a au même moment fait l'expérience de différentes techniques de développement narratif, notamment par l'intermédiaire du texte. Si cette forme de jeu textuel a évolué aujourd'hui en incluant d'autres éléments que la technique des années 1980 ne pouvait pas inclure, elle garde en commun avec la littérature expérimentale actuelle certains éléments qui nous invitent à interroger la limite entre ces deux médias.

\section{Du texte à l'hypertexte}

4 La création littéraire a connu dans la seconde moitié $\mathrm{du} \mathrm{xx} \mathrm{x}^{\mathrm{e}}$ siècle une série d'évolutions qui ont déterminé la direction que les auteurs allaient prendre. Si l'arrivée des nouveaux médias de représentation, comme la photographie et le cinéma, a remis en question la capacité qu'avaient tant la littérature que la peinture à dire le monde, c'est surtout le formalisme et le structuralisme qui ont changé la perception que l'on avait de l'auteur et de son œuvre. En effet, celle-ci devenait texte et l'auteur simple scripteur, considération qui s'est cristallisée, entre autres, dans l'article de Barthes qui remet en question la centralité du rôle de l'auteur jusqu'à en proclamer la mort symbolique (Barthes 1968).

\section{La littérature potentielle et la systématisation de la création}

Parallèlement à ce débat, on assiste à la création de l'Ouvroir de Littérature Potentielle en 1960 par François Le Lionnais et Raymond Queneau. Il s'agissait pour l'Oulipo de faire de la littérature « volontaire » (Lescure 1973 : 32), c'est-à-dire laisser le moins de marge possible au hasard de la création ${ }^{2}$. Pour ce faire, l'Oulipo propose de faire de la 
littérature à contrainte : chaque œuvre doit avoir été écrite selon un certain nombre de contraintes, formelles ou structurelles.

6 Les exemples de contraintes formelles les plus connus sont le lipogramme (l'écriture d'un texte sans utiliser une ou plusieurs lettres données), dont on connaît de Perec le roman La Disparition, ou encore la méthode dite lescurienne, qui consiste à remplacer certains mots par leurs voisins du dictionnaire (le plus connu est le $S+7$ : à chaque substantif, on le remplace par le septième substantif qui suit dans un dictionnaire donné). Queneau en a proposé un dans ses Exercices de style (1973a) sous le titre "Translations ». L'incongruité du résultat étonne d'autant plus que le processus de modification suit un ordre et des règles bien précis.

7 Mais c'est parmi les contraintes structurelles qu'on trouve les expérimentations les plus intéressantes pour notre propos. En effet, il s'agit de construire un texte selon certains principes de composition, là aussi liés aux mathématiques. C'est ainsi que Perec conçoit le plan de La vie mode d'emploi (1978), dont les chapitres, qui décrivent chacun une pièce différente d'un immeuble parisien, suivent le déplacement d'un cavalier sur un échiquier qui recoupe la structure de cet immeuble. De même pour L'art et la manière d'aborder son chef de service pour lui demander une augmentation (Perec 1973), dans lequel le récit prend, tour à tour, chaque embranchement d'un organigramme présentant toutes les solutions possibles au problème: "Quand et comment faut-il demander une augmentation à son chef de service?»

Ces recherches sur la structure narrative ont très tôt intéressé Italo Calvino qui, dans son article, tiré d'une conférence, «Cibernetica e fantasmi » (1980), montre les enjeux que pose une narration basée sur la combinatoire. Il retrace avant tout l'histoire du récit et imagine que les hommes des tribus n'avaient que quelques éléments qu'ils pouvaient mettre ensemble pour construire un récit, ce qui permet de mieux comprendre qu'une histoire n'est qu'une combinaison de certains éléments mis en contact, et que si le spectre des possibilités aujourd'hui s'est élargi, le fonctionnement de la construction d'un récit n'est pas différent. Calvino en déduit alors qu'une machine pourrait très bien, si la technologie en était capable ${ }^{3}$, écrire de manière totalement autonome des romans selon les goûts et les habitudes des lecteurs, ce qui permettrait de se débarrasser une bonne fois pour toutes de l'auteur. Même si ce discours n'est pas sans une certaine dose d'ironie (Calvino est convaincu du rôle essentiel de l'auteur) et que Calvino nuance son propos et montre les limites d'une telle perspective en réaffirmant l'importance du tabou, du non-dit, du «fantôme ", que la machine ne peut reproduire, on retient chez lui l'intérêt qu'il porte à la création littéraire comme ars combinatoria.

9 En témoigne sa production lorsqu'il entre en contact avec l'Oulipo. Outre certains exercices de style (poèmes en lipogrammes, composition d'un syllabaire illustré à la manière de Perec) et le roman représentatif de sa participation au groupe parisien, $\mathrm{Si}$ par une nuit d'hiver un voyageur (Calvino 1979), où le lecteur se retrouve emprisonné dans une succession de débuts de romans dont il ne peut jamais connaître la suite, Calvino s'est essayé à la composition narrative combinatoire.

Dans une nouvelle qui aurait dû donner lieu à un roman, "L'incendio della casa abominevole» (Calvino 1973b), le personnage principal, Waldemar, est un informaticien chargé de résoudre une enquête grâce à son ordinateur. Le problème posé par l'enquête est le suivant : une maison a brûlé avec tous ses habitants. Parmi les décombres, on retrouve un cahier sur lequel on peut encore lire une liste de crimes 
numérotés de 1 à 12 sans qu'ils soient imputés à l'un des quatre habitants de la maison. Il s'agit donc de reconstruire la suite des événements en sachant que, les quatre personnages pouvant générer douze types de relations différents (en effet, six couples sont possibles, et une action entre deux personnages peut être effectuée dans un sens ou dans l'autre), le nombre de combinaisons possibles s'élève à $12^{12}$, c'est-à-dire à plus de huit mille milliards de possibilités. Si ce n'est que Waldemar, pendant les longs moments de réflexion de la machine, commence à soupçonner son client, l'assureur Skiller, et le fait entrer dans l'équation, et se sentant menacé, s'ajoute lui-même dans le calcul. L'intrigue se complexifie donc, d'autant plus que les combinaisons possibles sont limitées par certaines contraintes, comme l'impossibilité d'un personnage de commettre un crime s'il l'a lui-même subi. Comme le raconte l'oulipien Paul Braffort sur son site internet (Braffort 2002), cette nouvelle a été utilisée par Calvino lors d'une journée d'études proposée par l'Oulipo autour du thème "Écrivains, ordinateurs, algorithmes » au Centre Pompidou le 15 juin 1977. On y voit l'intérêt que portait Calvino pour la narration combinatoire et pour l'emploi de l'informatique en littérature à une époque où l'ordinateur n'était pas encore d'accès aisét.

\section{L'objet-livre : renouvellement et dépassement}

11 En plus de mener une réflexion sur la logique du récit, cette période littéraire contribue à remettre en question la façon dont on voit l'objet livre, dans sa matérialité et dans son approche. Cortázar est sans doute l'un des cas les plus probants avec son roman Marelle (1967), écrit de manière à ce que le lecteur puisse choisir le chemin le plus rapide (lire les 56 premiers chapitres dans l'ordre jusqu'à la fin, signalée par trois étoiles) ou le chemin le plus long, en suivant un parcours proposé par l'auteur qui intercale entre chacun des chapitres principaux des chapitres secondaires qui viendront apporter des précisions sur certains personnages ou sur l'intrigue, ou qui proposeront des bribes de réflexions littéraires, philosophiques ou métaphysiques, enrichissant ainsi cette histoire d'intellectuels par les sujets de conversation qui les animaient dans leur appartement parisien. Cependant, cette disposition ne suit pas un ordre mathématique précis. Les pages des chapitres secondaires sont dans le désordre et l'auteur ne cherche pas à faire adopter à son lecteur un quelconque pattern de lecture. C'est surtout le livre dans sa linéarité qui est remis en question, ainsi que sa manipulation en tant qu'objet: le lecteur ne se contente plus de tourner les pages, mais s'adonne à un jeu d'enquête organisé par l'auteur.

Cette tendance, représentée ici par le roman de Julio Cortázar, se traduira par le passage de la littérature dans l'environnement numérique. On trouve dans les expérimentations de groupes comme l'Oulipo un intérêt certain pour cet outil encore émergent, si bien que la création en 1981 de l'Atelier de Littérature Assistée par la Mathématique et les Ordinateurs (Alamo) par Jacques Roubaud et Paul Braffort a surtout été le lieu d'applications informatisées d'exercices oulipiens déjà existants. Ainsi, le «Conte à votre façon » (Queneau 1967), dans lequel le lecteur a le choix entre trois possibilités à chaque étape $d u$ récit, s'est vu transposé dans une version numérique, et le programme qui en a permis l'élaboration a été laissé à la disposition des internautes pour qu'ils puissent à leur tour créer un texte interactif. Par la suite, certaines créations ont pris tout leur sens dans un environnement numérique. Le « Rimbaudelaire » (Roubaud 2008) est un mélange entre Le Dormeur du val de Rimbaud et le vocabulaire poétique baudelairien. Chaque substantif du sonnet est remplacé 
aléatoirement par un autre mais dans le respect tant de la cohérence du texte que de la métrique et des rimes du poème. Le lecteur peut ainsi en générer à sa guise et admirer le résultat.

13 Le site de l'Alamo contient toute une série d'expériences ludiques de ce genre, sans pour autant aboutir à un véritable projet de création narrative. L'arrivée de l'informatique, puis d'Internet, a en effet ouvert de nombreuses possibilités de construction de textes, mais on peut s'interroger sur ce qui constitue une avancée structurelle essentielle dans la création littéraire par rapport à ce qui n'apporte que ce que René Audet appelle un "effet de surface» (2015: 2). En effet, l'entrée de la littérature dans le numérique n'a pas eu lieu sans un certain flottement entre la technique et le texte lui-même, d'où son constat: "La surenchère du contrôle de l'expérience de lecture conduit à des hapax, où le rouage étouffe le sens » $(2015: 3)$.

14 L'arrivée $\mathrm{du}$ numérique a pu accentuer la nouvelle approche du texte en l'affranchissant des contraintes du format livre, même pour des œuvres qui, à l'origine, n'avaient pas été prévues pour cet usage. La Simulation humaine de Daniel de Roulet est une série de nouvelles et romans initialement écrits pour être lus au format classique. C'est une saga romanesque dont les volumes, qui peuvent être lus indépendamment les uns des autres, sont liés par la thématique de la science nucléaire et par des retours de personnages. C'est au terme de vingt années d'écriture que l'auteur a senti le besoin de structurer son œuvre de manière non linéaire. Il s'est donc associé à deux chercheurs, Cyril Bornet et Frédéric Kaplan, qui voulaient étudier la genèse d'un tel projet afin qu'ils l'aident sur l'aspect technique. Le constat auquel ils arrivent au commencement du projet recoupe la remarque de René Audet :

Du point de vue de la forme enfin, force est de constater que les contenus très novateurs publiés autour du thème du livre numérique n'ont pas apporté de changements radicaux à la lecture en général. (Bornet, Roulet, et Kaplan 2014 : § 16)

15 Ainsi, parmi les directions que les deux chercheurs pouvaient prendre pour insérer l'œuvre de Daniel de Roulet dans un environnement numérique, ils ont préféré restructurer les chapitres plutôt que les enrichir de documents annexes à la création littéraire, afin d'offrir au lecteur une immersion sans sources de distraction. En effet, il s'agissait avant tout de maintenir la fluidité de l'expérience de lecture numérique, et donc de minimiser la présence d'une interface qui viendrait faire de l'ombre au texte. De ce fait, la fin d'un chapitre ressemble à ceci : 
Fig. 1. La fin d'un chapitre dans La Simulation humaine de Daniel de Roulet

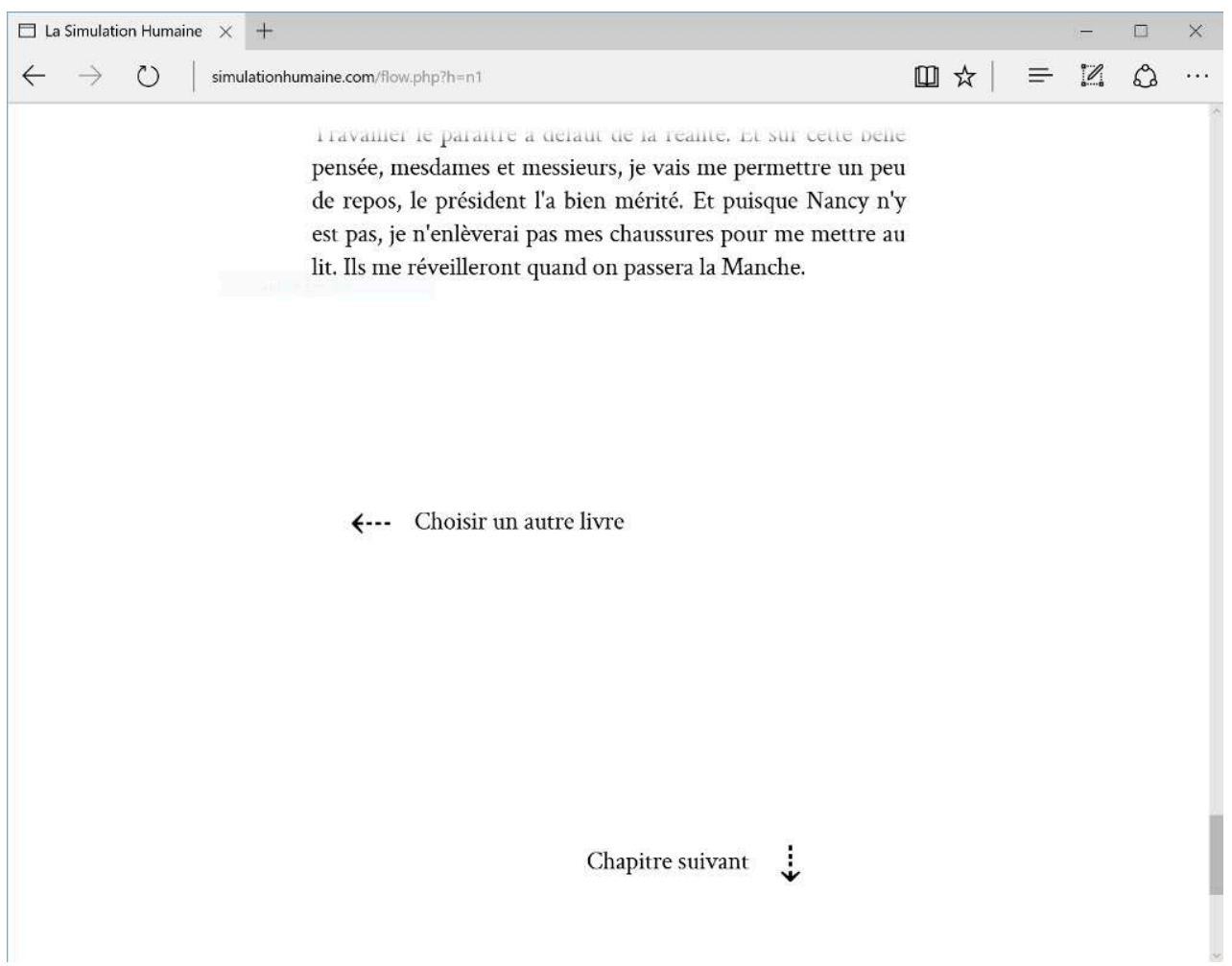

Si le lecteur ne veut pas interrompre sa lecture pour passer à un autre ouvrage, il lui suffit de faire défiler la page jusqu'au chapitre suivant. L'option «Choisir un autre livre" renvoie le lecteur à un sommaire classant les histoires selon leur longueur (nouvelle, roman court, roman long), ce qui permet une lecture par « butinage ${ }^{5}$ ». Pour tout marque-page, un compteur indique combien de chapitres ont été lus de toute la saga. Si cette adaptation numérique laisse au lecteur la liberté de commencer par n'importe quelle histoire et lui permet une lecture transversale de l'ensemble de la saga, l'œuvre ne perd pas sa dimension linéaire, héritée du format d'origine. En effet, quels que soient les choix du lecteur dans la lecture des différents romans et nouvelles, son parcours de l'œuvre ira toujours, bien que morcelé, du début vers la fin.

Le projet de Daniel de Roulet est bien plus récent que les expériences de l'Alamo, et pourtant c'est la même question sur l'apport d'une transposition numérique qui est posée : comme l'outil est nouveau, l'intérêt porte plus sur ce qu'il permet de faire que sur la signification que l'environnement numérique peut apporter. Il s'agit sans doute encore de ce que Janet Murray, dans Hamlet on the Holodeck, appelle "incunabula» ([1997] $2000: 29$ ), à savoir ces formes précoces d'un genre ou d'un médium aux traits encore hésitants. On peut se demander alors que faire de ces nouvelles possibilités offertes par l'informatique, que ce soit la vidéo et l'image numérique, le son intégré, et surtout les différents types de réseaux narratifs offerts par l'hypertexte.

18 Si les trois premiers éléments tendent à être considérés comme des ajouts illustratifs à moins d'étudier l'objet d'un point de vue intermédial ${ }^{6}$-, c'est le troisième qui permet au lecteur d'aborder le texte d'une façon radicalement différente.

La notion d'hypertexte a deux origines différentes. Gérard Genette, dans Palimpsestes (1982), réfléchit à la notion d'intertextualité en établissant un rapport entre un texte donné (l'hypertexte) et les textes auxquels il fait référence, plus ou moins 
explicitement, appelés les hypotextes. Parmi les différents types d'hypertextes, Genette identifie les "hypertextes autographes à hypotexte ad hoc ", c'est-à-dire des hypertextes dont "la version "originale" a été manifestement calculée pour donner lieu à la version seconde, à moins qu'un heureux hasard n'ait fourni les deux ensemble » (1982: 73). Comme exemple, Genette évoque les Cent Mille Milliards de Poèmes de Queneau (1961), recueil de dix sonnets dont chaque vers est permutable. Ainsi, le lecteur, qui doit manipuler dans un livre des languettes indépendantes, a face à lui une œuvre de poésie combinatoire dont les résultats possibles s'élèvent à $10^{14}$.

Si la conception de l'hypertexte de Genette dépasse l'idée de combinaison et de littérature potentielle, c'est dans l'acception de l'hypertexte autographe que l'on peut percevoir un écho à la version de Ted Nelson qui, en 1960, développe le concept d'hypertexte dans un contexte de naissance d'un réseau issu du projet Xanadu, précurseur d'Internet. Ici, «hypertexte » désigne le lien attribué à un élément textuel qui permet à l'utilisateur de passer d'un environnement à un autre. À l'origine, le projet était destiné à faciliter l'accès aux informations aux chercheurs des universités américaines, mais il n'a pas tardé à être utilisé aux États-Unis par des auteurs qui en ont fait une utilisation littéraire, à l'instar de Michael Joyce dans afternoon, a story (1987).

21 L'hypertexte revêt de nombreuses formes, mais il peut être défini selon deux structures majeures : en arbre ou en réseau.

Fig. 2. Exemples d'hypertextes en arbre et en réseau, ou « rhizome ${ }^{7}$

Hypertexte en arbre

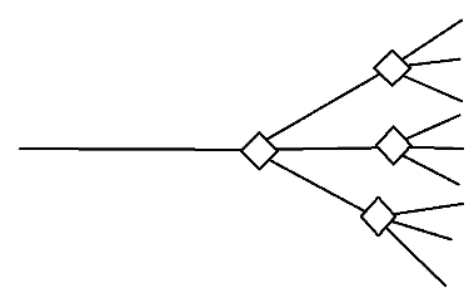

$\circlearrowright$ Fil narratif
Hypertexte en réseau

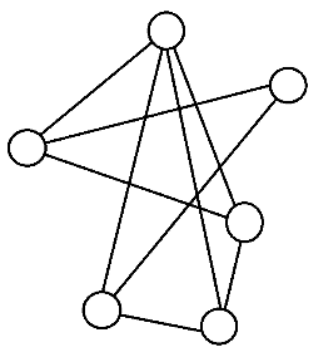

Nœud narratif

Jonctions narratives
22

Sur le schéma, la représentation de l'hypertexte en arbre rappelle, par sa structure fractale et tripartite, le « Conte à votre façon » de Queneau, mais ce qui importe, ce sont les notions de bifurcation et de hiérarchie. Ainsi, on peut voir dans un roman comme Marelle une structure hypertextuelle en arbre, car d'un tronc narratif commun partent des digressions qui ramènent toujours le lecteur au fil principal. En revanche, afternoon, a story relève de la structure rhizomique. Le roman, qui raconte l'histoire d'un homme se demandant si l'accident de voiture qu'il avait vu le matin en se rendant au travail était celui de sa femme et de sa fille, est un ensemble de 539 pages-écrans reliées entre elles par plus de 900 liens. Il existe une vingtaine de débuts, et, surtout, il n'y a pas de fin. Si le lecteur se contente d'appuyer sur la touche «Entrée » à la fin de chaque page, 
l'histoire s'interrompra au bout de quelques dizaines d'écrans. Pour une véritable lecture de l'hypertexte, il devra cliquer sur des liens cachés dans certains mots. Mais, comme l'explique Jean Clément dans l'analyse qu'il a faite du récit :

On comprend dès lors qu'il s'agit d'un récit labyrinthique, comme ces jardins du Moyen Âge conçus pour que l'on s'y perde dans le dédale des allées bordées de haies. Avec quelques différences cependant. Tandis que les labyrinthes du Moyen Âge étaient faits d'arbustes indéracinables, le labyrinthe hypertextuel est en perpétuelle métamorphose. En repassant par les mêmes lieux on les trouve changés, méconnaissables. Ils ne débouchent plus sur les mêmes suites de parcours. (Clément 1994a)

En effet, certains liens ne sont accessibles au lecteur qu'après son passage par certaines pages. Il faut donc apprendre à se déplacer dans l'hypertexte, mais, comme le début n'est jamais le même, il sera impossible pour deux lecteurs d'avoir la même expérience de lecture. «L'œuvre totale, conclut Jean Clément, ne peut être que virtuelle » (1994a). Il ajoute que l'expérience de lecture que ce format induit relève plus de l'errance poétique que de l'implication narrative. De cette manière, l'histoire s'achève quand le lecteur cesse de parcourir l'hypertexte, plus par lassitude que par satisfaction. C'est, selon Renée Bourassa, le problème des productions d'Eastgate Systems (la maison d'édition d'afternoon, a story) qui, voulant remettre en question les attentes narratives de leurs lecteurs, finissent par les perdre :

Substituer une déambulation aveugle agissant par collage aléatoire au processus créateur de l'auteur a toutes les chances de mener à de piètres agencements; ces fragments se perdent dans la redondance et mènent, finalement, à l'échec de

l'expérience de lecture. (Bourassa 2010 : 35)

Dès lors, comment faire pour garder l'attention du lecteur tout en lui offrant une possibilité de co-construction narrative ? Ce qu'on constate, dans chacun des exemples qu'on a vus jusqu'à présent, c'est que le trait dominant et structurant de ces fictions interactives était le temps. Autrement dit, alors que le lecteur est invité à naviguer dans un espace narratif, le résultat de cette navigation ne diffère pas d'un récit linéaire, avec ses intrigues, ses prolepses et ses analepses, à ceci près que la construction qui soustend le tout, dont la cohérence était auparavant assurée par la figure de l'auteur, devient une sorte de jeu de hasard auquel il appartient au lecteur de donner du sens.

Cela ne signifie pas que la littérature n'a pas trouvé sa place dans l'environnement numérique : des maisons d'édition comme Quintadicopertina (Gênes) publient des ebooks hypertextuels, allant du roman interactif au recueil de poésies utilisant les possibilités versatiles d'un écran ${ }^{8}$. Mais, de l'avis même de son co-fondateur, Fabrizio Venerandi ${ }^{9}$, la littérature électronique reste trop souvent cantonnée à une simple transposition de la littérature papier sur l'écran, sans exploiter les nouvelles possibilités de l'environnement hypertextuel et hypermédiatique.

\section{Jeu vidéo et hypertexte}

Si la littérature a fait son entrée dans le numérique en adoptant certains de ses codes, un autre média né avec la culture numérique a absorbé très tôt certaines logiques narratives propres aux expérimentations littéraires citées plus haut. Cependant, il a contribué à instaurer un paradigme narratif non plus basé sur le temps, mais sur l'espace. 
27 Ajoutons, en préambule, que la narration dans le jeu vidéo a longtemps été vue par le prisme du débat entre ludologues et narratologues, les premiers reprochant aux seconds de vouloir plaquer la narratologie et les études littéraires sur un objet autre, et proposant plutôt de fonder de nouveaux outils d'analyse ${ }^{10}$. Ce débat a trouvé un équilibre dans les propos de Henry Jenkins qui préfère voir le game design comme une architecture narrative (2004).

\section{Le jeu textuel et la systématisation du schéma actantiel}

En 1976 sort le jeu Collossal Cave Adventure (Crowther 1976), un jeu d'aventure textuel qui entend reprendre et automatiser le principe du jeu de rôle papier, même si cela signifie restreindre les possibilités narratives offertes par ce dernier ${ }^{11}$. Il s'agit d'une sorte de dialogue avec un narrateur qui dit au joueur où il se trouve et lui demande quelle action il veut accomplir: se déplacer (aller au sud, au nord, etc.), prendre ou utiliser des objets, ouvrir des portes, prononcer une formule magique. Pour toute interaction, le joueur dispose d'une boîte de dialogue dans laquelle il entre des formules simples en attendant la réaction du narrateur. Si la formule n'est pas reconnue dans la situation donnée, il recommence avec une autre. Cette initiative s'inscrit dans un contexte d'exploration de l'informatique, notamment dans les laboratoires du MIT où l'intelligence artificielle est employée à différentes expériences, notamment ELIZA ${ }^{12}$ et Zork, un jeu d'aventure au fonctionnement proche du programme de William Crowther.

À propos de ces premières expériences interactives, Renée Bourassa en reconnaît l'attrait ludique tout en déplorant l'approche narrative qui se base sur des «prémisses esthétiques souvent naïves ou des schémas actantiels stéréotypés ». Cependant, ajoutet-elle :

[...] l'approche de cette génération d'informaticiens a ouvert de manière heuristique les potentialités de l'informatique pour créer de nouveaux environnements narratifs. Ceux-ci tiennent compte à la fois des questions ergonomiques et des diverses modalités de manipulation de l'information (bases de données, outils de recherche, etc.). Leur exploration a généré des interfaces beaucoup plus intuitives, qui dépassent de loin, sur le plan de l'interactivité, la simple navigation des hyperfictions textuelles. (Bourassa $2010: 41$ ) 


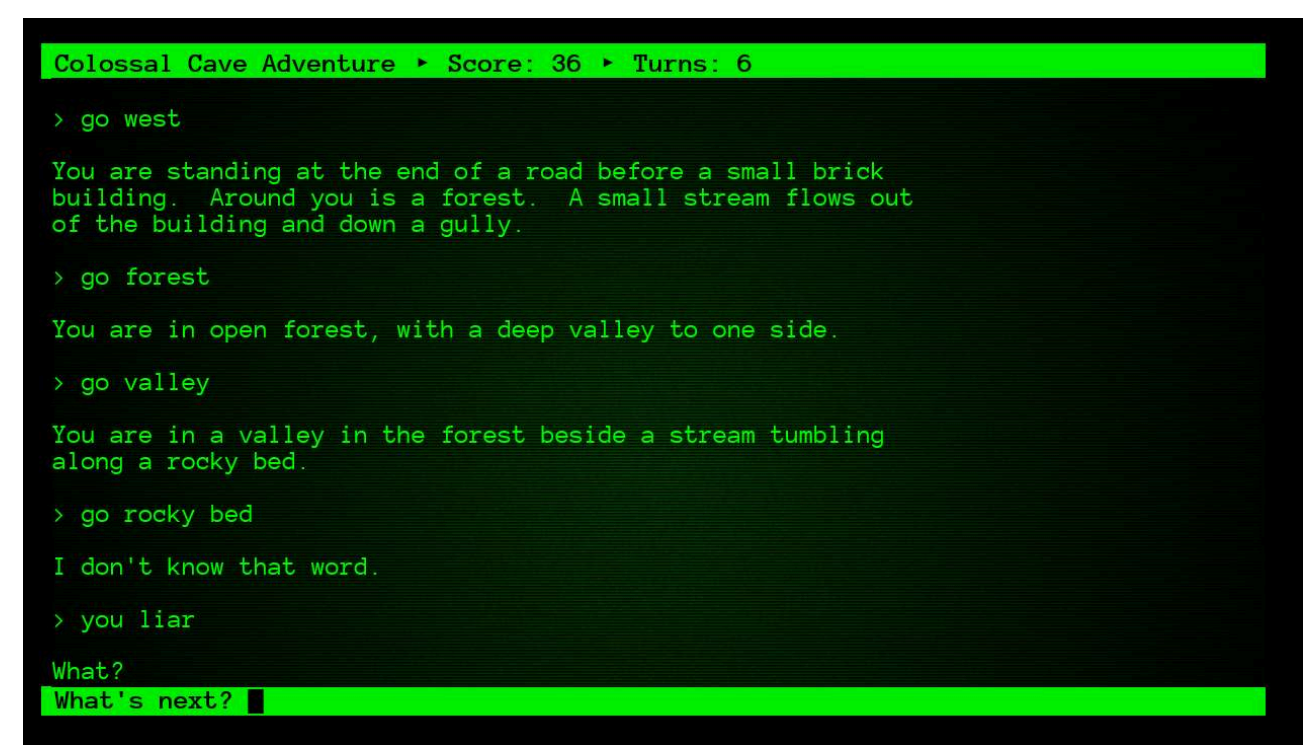

En regardant une capture d'écran de Colossal Cave Adventure, on se retrouve face à un dialogue entre l'utilisateur et la machine. Le narrateur donne à l'utilisateur des indications spatiales pour indiquer autant de lieux où ce dernier peut se rendre quand il le souhaite. Ce sont les objets qu'il utilisera au bon moment qui feront avancer l'histoire, le but étant de trouver une grotte remplie de trésors après avoir résolu des énigmes et traversé des labyrinthes. Dans les productions suivantes, le fait d'entrer des verbes au clavier sera remplacé par une liste d'actions sur lesquelles il faudra cliquer, et les indications textuelles laisseront la place à des décors dessinés à l'ordinateur dans lesquels le personnage du joueur pourra se déplacer. Le jeu d'aventure textuel donnera ainsi naissance à ce qu'on appelle encore aujourd'hui le point-and-click ${ }^{13}$.

La particularité de ces œuvres, par rapport aux fictions hypertextuelles que nous avons vues précédemment, réside dans leur dimension narrative fortement spatialisée. Chaque intervention du narrateur dans Colossal Cave Adventure est en fait une description de ce qui entoure le lecteur-personnage. Cela explique pourquoi le genre du "jeu de quête» (Bourassa 2010: 133) a connu une conversion vers l'image aussi efficace.

32 Et pour cause : la métaphore spatiale constitue, pour Renée Bourassa, une solution privilégiée pour permettre à l'utilisateur de naviguer dans un environnement hypertextuel (puis, avec l'arrivée du son et de l'image, hypermédiatique) : «Le monde diégétique s'incarne dans un espace navigationnel qui en favorise la saisie intuitive, la lisibilité et l'intelligibilité » (Bourassa 2010 : 147). À partir de quoi, l'interactivité ne consiste plus, pour l'utilisateur, à composer son histoire en tirant chaque partie comme les cartes d'un paquet, mais de se déplacer dans un espace fictionnel et, par ses déplacements, d'actualiser une potentialité narrative que cet espace contenait.

\section{Du texte à l'image}

33 L'un des exemples, devenus archétypes, de cette exploration narrative de l'espace fictionnel, est le jeu de quête Myst (Cyan Worlds 1993), un jeu d'exploration en vue subjective dans un univers fantastique représenté au joueur par des images fixes. 
«Myst » est le nom de l'île sur laquelle le joueur, incarnant un personnage sans nom (surnommé "l'Étranger »), se retrouve grâce au "livre de liaison", qui permet de passer d'un monde à l'autre. Si le jeu a marqué les esprits à sa sortie et qu'il est toujours cité, aujourd'hui, comme un classique, c'est tout d'abord pour l'aspect visuel qui, pour le début des années 1990, était impressionnant de réalisme, mais aussi parce qu'il a su montrer au grand public que le jeu vidéo était capable de produire plus que des jeux de tir ou de plate-forme ${ }^{14}$, donnant naissance à un genre de jeu moins axé sur la performance et plus sur la contemplation et la réflexion.

La nouveauté de ce jeu est aussi le fait que le joueur n'est plus un lecteur, mais parcourt l'île en la voyant, ce qui rend plus immédiate l'approche de l'environnement. Le texte se retrouve dans les documents que le joueur trouve pendant sa quête, qu'il s'agisse de lettres, de livres, ou, dans les suites du jeu, de dialogues joués par des acteurs. En somme, le texte ne disparaît pas, mais se retrouve transposé dans un contexte plus défini et visuel. Les éléments textuels - ou scripturaux - qu'on ne retrouve plus sont d'un côté les descriptions, et de l'autre les actions, décrites ou accomplies, qui sont retranscrites visuellement à l'écran.

L'évolution du jeu vidéo allant de pair avec l'évolution des technologies, les environnements fictionnels virtuels sont devenus de plus en plus convaincants de réalisme. L'un des passages les plus frappants a été celui de la succession d'images fixes (à la manière de Myst) vers l'évolution du joueur dans un environnement en trois dimensions généré en temps réel. Pendant longtemps, les deux pratiques ont coexisté parce que chacune avait ses avantages et ses inconvénients : la première donnait à voir des paysages plus riches, alors que la seconde permettait une performance ludique plus variée, au détriment de l'aspect visuel grossier ${ }^{15}$. Puis le jeu de quête a fini par adopter les décors générés en temps réel, amplifiant ainsi l'expérience narrative liée à l'exploration de l'espace fictionnel.

Aujourd'hui ${ }^{16}$, les héritiers de Myst sont appelés des "walking simulator", des simulateurs de promenade. La formulation peut surprendre, mais elle a le mérite de mettre l'accent sur l'importance que revêt l'environnement fictionnel dans l'expérience narrative et esthétique. Dans ces jeux, il est rarement question d'affrontements, de points d'expérience, et la notion de gagner ou de perdre est souvent reléguée au second plan. Gone Home (The Fullbright Company 2013), par exemple, se passe entièrement dans une maison. Une étudiante rentre aux États-Unis après une année passée en Europe et, comme personne n'est là pour l'accueillir, elle parcourt la maison à la recherche d'indices pour comprendre ce qu'il s'est passé. Le joueur devra donc accéder à divers documents (du livre au post-it en passant par la cassette audio) qu'il trouvera dans les meubles de la maison, afin de découvrir l'intrigue. Dans The Vanishing of Ethan Carter (The Astronauts 2014), le joueur incarne un détective privé qui recherche un enfant dans la "Red Creek Valley». Dans une ambiance de thriller, le joueur parcourt cette vallée pour recueillir des indices dans les différentes localités. Dans les deux jeux précédemment cités, une voix off accompagne les pas du personnage : il s'agit du monologue intérieur du protagoniste qui fait part de ses réflexions au joueur.

37 On voit bien dans ces deux exemples l'idée, d'une part de monde fictionnel structuré dans un espace défini, et, d'autre part, celle d'errance qui caractérise l'expérience narrative du joueur. Nous parlons en effet d'errance, mais elle n'est pas du même ordre que l'errance qui caractérisait la lecture d'un hypertexte comme afternoon, a story. Ici, la 
métaphore spatiale qui organise les informations relatives au monde fictionnel permet une meilleure appréhension de ce que Jean Clément appelait « l'œuvre totale » : certes, il est impossible d'englober d'un regard toute l'œuvre, mais la vue de la maison dans Gone Home ou le panorama de "Red Creek Valley » dans The Vanishing of Ethan Carter permettent au joueur d'avoir une vision cohérente de l'univers diégétique dont il s'apprête à examiner les détails.

Cela ne veut pas dire que le "jeu de quête", ou aujourd'hui plus précisément le " walking simulator ", s'est conformé à un seul type de narration basé sur une enquête de type policier et qu'il n'y a aucune alternative. Bientôt l'été (Tale of Tales 2013) prend même ce modèle à contre-pied en invitant d'emblée le joueur à une expérience gratuite. Comme le cite Erwan Higuinen dans son compte rendu du jeu (2013) :

Ceci n'est pas un jeu où l'on gagne. Jouez juste pour l'expérience. Promenez-vous, déambulez et contemplez. Il n'y a aucun but. Il n'y a aucune histoire. Laissez-vous porter par l'atmosphère. Ne pensez à rien. N'aspirez à rien. Ne soyez que vousmême.

Le joueur se contente de se promener sur une plage et peut discuter avec un personnage féminin en utilisant des phrases issues des œuvres de Marguerite Duras, dont le jeu est inspiré. De cette manière, les développeurs évacuent définitivement les codes habituels du jeu vidéo, voire du jeu en général : ni ludus - si ce n'est les règles du monde fictionnel et les limites d'action qu'il impose au joueur - ni paidia ${ }^{17}$, car le joueur n'est pas porté par une quelconque pulsion ludique mais, au contraire, est invité à prendre une posture contemplative.

\section{Spectre ou limite?}

40 À partir de là, il nous faut nous poser la question de ce qui différentie ces deux médias, maintenant que l'environnement dans lesquels ils se trouvent a contribué à brouiller la distinction claire qui les séparait. En d'autres termes, qu'est-ce qui distingue la littérature numérique et expérimentale du jeu vidéo narratif?

\section{Une affaire de forme}

41 La première réponse qui nous vient à l'esprit réside dans le constat d'une différence formelle entre les deux. Alors que le jeu vidéo est quelque chose que l'on regarde comme son nom l'indique - la littérature reste liée à son aspect scriptural, et son interacteur est avant tout un lecteur. Dans ce cas, la littérature produirait un discours uniquement par l'intermédiaire de la parole écrite, là où le jeu vidéo emploierait différentes formes médiatiques, laissant le texte de côté. Ainsi, comme on l'a vu, les paragraphes descriptifs sont remplacés par les éléments décrits donnés à voir au joueur, et les actions sont retranscrites à l'écran. Il ne resterait du texte écrit qu'un reliquat esthétique servant à exprimer ce que l'image ou le son ne peuvent retranscrire.

Cette distinction appelle plusieurs objections. Tout d'abord, même si les jeux d'aventure textuelle se sont affranchis, comme on l'a vu, de la médiation écrite pour donner à voir au joueur l'univers fictionnel dans lequel il évolue, cela ne veut pas dire que cette forme a disparu. Le jeu de Zoe Quinn, Depression Quest (2013), en est un exemple. 
Il s'agit d'un jeu à interface textuelle anglophone dans lequel le joueur incarne un homme atteint de dépression et pour qui chaque événement de la vie quotidienne (partir au travail, aller se coucher, rendre service à une connaissance) est une épreuve pour le joueur l'incarnant qui doit choisir, après chaque paragraphe narratif, une action parmi plusieurs propositions. Selon le choix du joueur, la situation présentée évoluera dans un certain sens et déterminera la suite de l'histoire. Sous chaque épisode raconté, figurent des cases indiquant le niveau de dépression du personnage, s'il voit un médecin et s'il suit un traitement. Plus l'état est grave, plus les cases sont brouillées, comme un écran de télévision qui reçoit mal le signal.

Fig. 4. La deuxième page de Depression Quest et le premier choix laissé au joueur

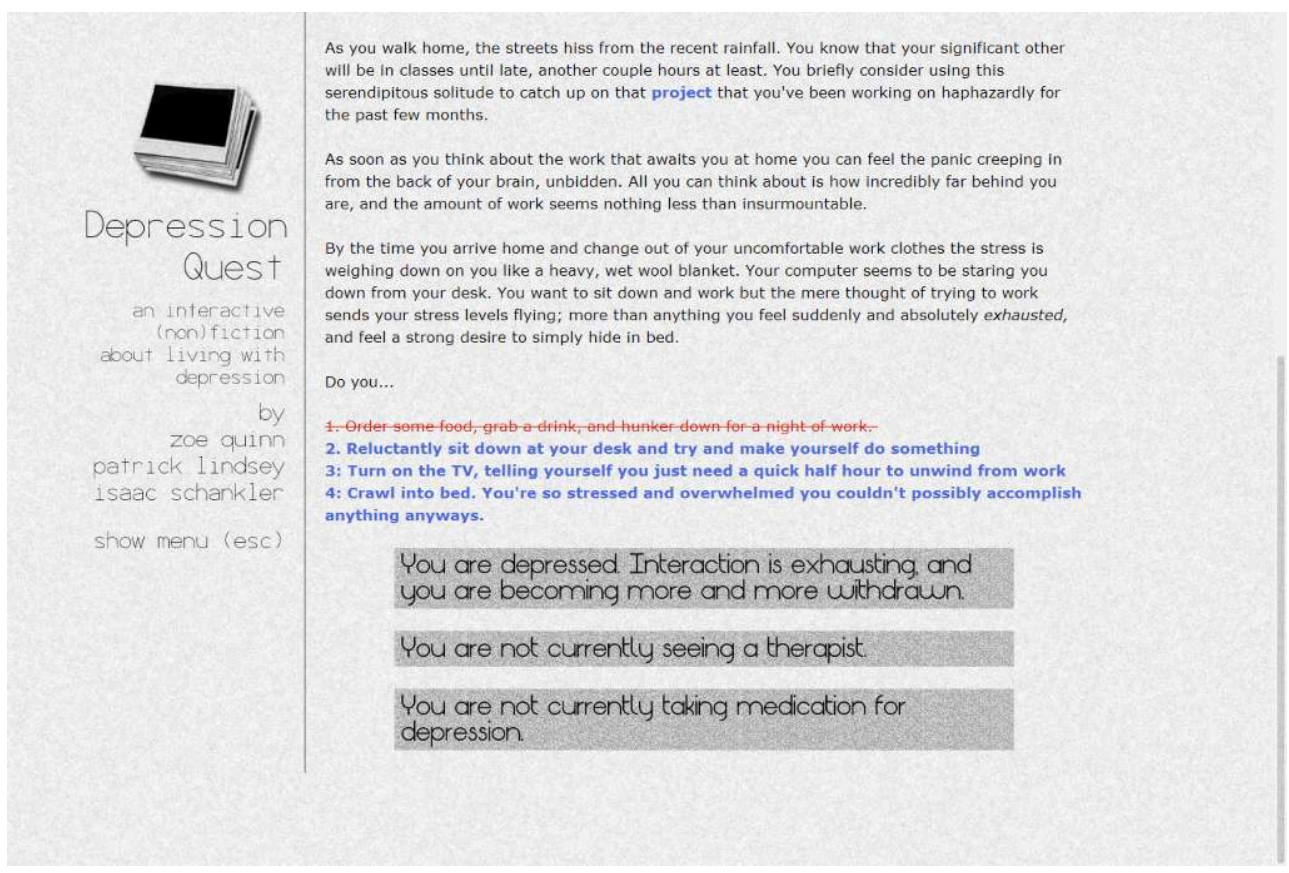

Après un incipit qui présente au joueur le personnage et son entourage, la deuxième page met en scène un retour à la maison après une journée de travail. Le personnage qui, dans la narration, est désigné par la deuxième personne " you » - ressent le stress de la journée et se sent par conséquent incapable de décider quoi faire. C'est alors que le jeu donne la main au joueur, par le biais des quatre liens hypertextes dont le premier est d'emblée rayé et écrit en rouge. Pourtant, l'option barrée ("Commander à manger, se prendre à boire, et s'installer pour une soirée de travail $\left.{ }^{18} »\right)$ semble être la plus directe pour résoudre la situation proposée, tant dans le contenu que dans la formulation (verbes d'action, syntaxe simple, rythme de la phrase qui semble souligner l'évidence de l'acte), alors que les suivantes sont de plus en plus laborieuses et donnent une impression d'immobilisme. La suivante, par exemple (" À contrecœur, s'asseoir au bureau et essayer de se forcer à faire quelque chose ") semble entravée par l'adverbe initial et les verbes enchâssés dont le seul objet dénote une certaine indétermination.

En fait, cette première série de choix a un but doublement pédagogique. Tout d'abord car il laisse entendre au joueur que certains choix seront barrés tout au long de la narration, et que cette mécanique sera un élément à prendre en compte dans l'expérience interactive. Ensuite parce que le jeu a pour but de sensibiliser l'utilisateur à ce qu'est la dépression. Ici, si une action qui semble aussi simple est rendue 
impossible, c'est pour faire comprendre au joueur que dans cette histoire, les décisions les plus simples ne seront pas nécessairement possibles, comme c'est le cas chez une personne atteinte de dépression.

Il faut ajouter que le jeu de Zoe Quinn a reçu un accueil mitigé de la part de la communauté de joueurs. Nous laisserons de côté l'affaire du Gamergate ${ }^{19}$ pour nous concentrer sur le fait qu'il est discutable de considérer cette œuvre comme un véritable jeu. L'hypertexte de Zoe Quinn suit un but de sensibilisation à la dépression qui rapproche plus le jeu du serious game, du jeu sérieux, dont la nature de jeu est constamment remise en question, par exemple lors de cette discussion entre Geoffrey Rockwell et Kevin Kee en 2009 qui donne lieu à un véritable débat socratique ${ }^{20}$. Ce jeu prolonge donc la question.

a question qu'on se pose ici regarde la nature de cette création. En quoi est-ce plus un jeu vidéo qu'un texte littéraire? Depression Quest est disponible gratuitement sur Internet, mais il peut être aussi téléchargé sur la plate-forme "Steam », spécialisée dans la vente de jeux vidéo. Par ailleurs, l'auteur n'en parle que comme « une (non) fiction interactive ", et, à l'exception de l'état du personnage présent sous le texte, peu d'éléments formels différencient l'œuvre de Zoe Quinn d'un hypertexte comme afternoon, a story de Michael Joyce.

La seconde objection est que la notion de texte, d'écriture, peut s'appliquer au jeu vidéo, quelle qu'en soit sa forme : pour reprendre le mot de Ian Bogost, le jeu vidéo est un « artefact procédural » (2006: XIII), c'est-à-dire un objet qui contient dans son code toutes les interactions qui peuvent avoir lieu. Ce code, n'est composé que de texte, bien qu'informatique, si bien que Jean-Paul Fourmentraux, à l'occasion d'un colloque sur les formes audiovisuelles connectées ${ }^{21}$, faisait remarquer que la création numérique, relevant essentiellement d'un art de la programmation, "rapproche l'image d'une culture de l'écrit ». Marie-Laure Ryan (2015) effectue d'ailleurs le cheminement inverse lorsqu'elle désigne la littérature comme une forme de réalité virtuelle. La notion de texte est donc brouillée par ces nouveaux systèmes de signification, à tel point que Renée Bourassa propose, à la suite de Michel Fayol (1985) et de Patrick Colm Hogan (2003), tend à considérer jeu, récit, littérature, comme les traductions d'une «structure cognitive commune » qui « gère l'action du sujet au sein d'un environnement comme sa mise en récit du monde ». Avant d'ajouter :

Les notions d'intentionnalité, de motivation, de but et de quête interviennent soit dans le contexte d'un discours temporalisé (récit littéraire et filmique), soit dans celui d'une simulation spatiale qui plonge l'acteur humain en son cœur (jeu interactif). (Bourassa $2010: 138$ )

49 Ainsi, il est difficile de démêler le textuel du non textuel. Tout d'abord parce que ce qui, à un niveau de perception, apparaît comme non textuel, est en fait sous-tendu par une structure textuelle qui le définit. Ensuite parce que texte écrit et environnement hypermédiatique dans lequel on évolue via un avatar peuvent être considérés comme l'expression d'une même réalité cognitive dans un langage différent. Faut-il alors parler, plutôt que d'une limite à identifier entre les deux médias, d'un spectre qui aille de l'œuvre littéraire au jeu vidéo ? Mais, dans ce cas, si l'importance du texte écrit dans une œuvre donnée ne peut pas constituer un critère valable pour établir une distinction et un classement sur un éventuel spectre, quel pourrait être l'élément structurant? 


\section{Le spectre de l'interactivité}

50 Jusqu'ici, nous avons tenté d'établir une différenciation des œuvres en fonction de la manière dont elles ont été construites et avons partiellement négligé un élément qui pourrait nous permettre d'apporter un début de réponse.

51 Toutes ces œuvres, qu'il s'agisse des jeux vidéo, des fictions interactives, des hypertextes de fiction, ou même des poésies combinatoires oulipiennes, ne sont réduites qu'à leur existence potentielle si elles ne sont pas au contact d'un interacteur. Et même : tout texte (dirait-on) "purement » littéraire ne peut rester qu'à un état incomplet s'il n'est pas parcouru, compris, interprété, par un lecteur. Ainsi, chaque œuvre, vidéoludique ou littéraire, est à considérer comme interactive. Mais il serait maladroit de considérer que l'interactivité à l'œuvre dans un roman du XIX ${ }^{\mathrm{e}}$ siècle est comparable à celle dans un jeu de quête à la manière de Myst.

Eric Zimmerman (2004), cité par Gaëlle Debeaux dans sa thèse sur la stéréométrie littéraire (2017), identifie pour cela quatre niveaux d'interactivité :

- cognitive (l'activité interprétative impliquée par tout acte de lecture) ;

- fonctionnelle, (les gestes et les actions de l'utilisateur sur l'objet contenant le récit, sans que ces actions n'altèrent l'état du texte) ;

- explicite (la prise en compte des actions de l'utilisateur si bien que l'état du récit est modifié) ;

- méta-interactive, (tout ce qui relève de la création à partir de l'œuvre mais en dehors de celle-ci) $^{22}$.

Ainsi, une œuvre littéraire demanderait de la part du lecteur une interactivité fonctionnelle (il doit tourner les pages) et une interactivité cognitive. À ce sujet, dans La lecture comme jeu, Michel Picard porte une attention particulière à l'interactivité fonctionnelle dans l'acte de lecture : « Le mouvement des yeux, les saccades du regard, les mains qui poussent les pages en une sorte de nage minuscule [...]» $(1986: 155)$. Cela étant, force est de constater qu'elle est limitée.

$\mathrm{Au}$ contraire, l'interactivité fonctionnelle demandée par un roman comme Marelle tend à transformer le lecteur en un chercheur qui feuillette les pages d'une encyclopédie pour recouper des informations. On pourrait même considérer qu'il y a une part d'interactivité explicite dans le roman de Cortázar, dans la mesure où le lecteur peut altérer l'état du récit par son choix de suivre ou non les chapitres secondaires.

C'est au niveau de l'hypertexte de fiction que l'état du récit se retrouve le plus modifié par l'action du lecteur. afternoon, a story reste ici édifiant, surtout quand on pense que chaque expérience narrative est différente du début à la fin, et qu'il n'est pas possible d'avoir une vision globale de l'œuvre. En revanche, un jeu comme Myst comporte à la fois une possibilité d'interactivité fonctionnelle (pouvoir se déplacer dans un univers fictionnel, de manipuler des objets, d'agir sur l'environnement virtuel), et ce sont les actions du joueur qui déterminent l'avancement du récit, mais pas autant que dans l'hypertexte de Michael Joyce car le récit restera le même, quel que soit le temps mis à résoudre les énigmes et l'ordre dans lequel le joueur explore les lieux.

Renée Bourassa procède à ce genre de distinction pour comparer Doom et Myst :

Dans certains jeux comme Doom, la performativité s'active à partir des réactions physiques du joueur; celui-ci s'échappe temporairement du mode symbolique et oublie le contexte fictionnel pour s'abandonner aux vertiges de la jouissance ludique, dans l'immédiateté de la situation expérientielle. Mais le joueur demeure 
lui-même, il ne s'identifie pas à un personnage fictionnel. D'autres jeux comme ceux de la série Myst, où la prégnance du contexte fictionnel est plus grande, mettent davantage l'accent sur la dimension symbolique: ils penchent alors vers la narrativité et mettent en œuvre les mécanismes d'identification fictionnelle, selon une esthétique de la transparence qui cherche à effacer la présence de l'interface. Les deux attitudes ludiques coexistent de manière hétérogène. (2010:120)

Autrement dit, pour reprendre la taxinomie définie plus haut, Doom est un jeu comportant une grande interactivité fonctionnelle, mais dont l'interactivité cognitive et explicite sont limitées. Au contraire, Myst réduit grandement son niveau d'interactivité fonctionnelle tout en donnant une place plus importante à l'interactivité cognitive.

58 Il serait alors possible de jauger ces trois niveaux d'interactivité pour chaque œuvre afin de déterminer sa position dans un spectre qui irait de la littérature au jeu vidéo. Que cette classification - qui n'aurait rien d'une hiérarchisation - puisse apporter quelque chose dans la compréhension des œuvres ou non, elle aurait le mérite de mieux comprendre l'éventuelle limite qui séparerait littérature et jeux vidéo.

\section{Conclusion et perspectives}

Nous avons tenté de montrer dans cet article que la frontière qui sépare littérature et jeu vidéo n'était pas aussi nette qu'on ne le pensait. D'une part parce que la littérature au $\mathrm{xx}^{\mathrm{e}}$ siècle a connu une évolution liée à l'apparition de l'informatique; d'autre part car le développement de l'environnement informatique a permis l'émergence d'un nouveau médium qui a su absorber certaines logiques esthétiques propres à la littérature.

60 Tout le courant de la littérature potentielle - jusqu'aux oulipismes non liés à l'Oulipo a contribué à élargir le discours littéraire à l'environnement numérique, quitte à changer de but et à dérouter son lecteur. Mais, surtout, cet élargissement ne s'est pas fait sans une certaine convergence avec les expérimentations ludiques d'abord textuelles, puis fortement liées à l'image.

61 Dès lors, une distinction liée à la forme du médium n'est plus pertinente, tant la littérature et le jeu vidéo ont connu des mutations formelles qui ont contribué à brouiller ces distinctions. C'est pourquoi nous proposons l'établissement d'un spectre qui reposerait sur les différents niveaux d'interactivité (parmi lesquels, rappelons-le, l'interprétation esthétique et narrative) énoncés par Eric Zimmerman.

62 Cette classification ne peut pas ne pas connaître des exceptions, mais c'est sans doute le propre des systèmes taxinomiques que de voir certaines œuvres leur échapper. Plus que de répondre à un besoin de classer et différencier les œuvres et les médias, cette proposition nous permet de poser plusieurs conclusions et perspectives.

63 La première est que l'on peut envisager, à ce titre, l'apparition d'une littérature non scripturale. Un auteur, muni des bons outils, pourrait créer une expérience narrative visuelle, sonore, interactive, sans que le support principal soit le langage écrit. Le style d'un auteur serait retranscrit dans le choix des formes et des couleurs, les figures poétiques et rhétoriques reprendraient une syntaxe proche de ce qui se fait dans la bande dessinée et le dessin humoristique, et le discours narratif n'en serait pas moins fluide. La différence avec le cinéma résiderait justement dans les possibilités interactives offertes par cet hypertexte qu'il appartiendrait à l'utilisateur d'explorer de 
la même manière qu'on explore aujourd'hui un texte littéraire. Reste la question: s'agirait-il toujours de littérature?

Car la deuxième conclusion à laquelle nous sommes arrivés est une question de terminologie. Outre la question de savoir si la littérature numérique, interactive, voire non scripturale, serait encore de la littérature, il convient de s'interroger sur la pertinence à parler de « jeu vidéo » terme bien vague pour ce qu'il désigne. En parlant de jeu vidéo, on parle à la fois d'œuvres comme Myst, Doom ou même Tetris; on parle de simulateurs de vol servant à former des pilotes, d'applications pour passer le temps dans le métro, voire d'outils thérapeutiques ou pédagogiques. Mis à part un certain « air de famille» (Wittgenstein 1953), chacun de ces logiciels ne propose pas la même finalité, la même expérience esthétique, et ne s'adresse pas au même public. Sans compter que les niveaux d'interactivité sont radicalement différents d'un jeu à l'autre. Le jeu vidéo gagnerait donc à être mieux défini et le spectre que nous proposons pourrait faciliter cette redéfinition.

Enfin, cette étude nous confirme que l'émergence d'un nouveau médium provoque toujours une crise parmi les médias existants, lesquels passent ainsi par un processus de redéfinition, comme c'était le cas, par exemple, du théâtre face à l'arrivée du cinéma (Abirached 1978). Sans parler d'une « nouvelle chance » offerte à la littérature, comme a pu le faire Jean Clément (1994b : 36), étudier le jeu vidéo et son rapport à la littérature nous permettrait de comprendre comment la littérature a su se redéfinir et se réaffirmer en réaction à l'arrivée de ce nouveau paradigme.

\section{BIBLIOGRAPHIE}

Abirached, Robert, 1978, La Crise du personnage dans le théâtre moderne, Paris, Gallimard.

Audet, René, 2015, «Écrire numérique : du texte littéraire entendu comme processus », Itinéraires. Littérature, textes, cultures, $\mathrm{n}^{\circ}$ 2014-1, [En ligne], http://journals.openedition.org/ itineraires/2267, consulté le 14 juin 2018.

DOI : 10.4000/itineraires.2267.

Audureau, William, 2014, «Derrière le \#GamerGate, une nébuleuse antiféministe ", Le Monde.fr, 15 septembre 2014, rubrique Pixels, [En ligne], http://www.lemonde.fr/pixels/article/

2014/09/15/derriere-le-gamersgate-un-groupe-antifeministe_4485191_4408996.html, consulté le 14 juin 2018.

Barthes, Roland, [1968] 1984, « La mort de l'auteur », dans Le Bruissement de la langue, Paris, Seuil. Bogost, Ian, 2006, Unit Operations. An Approach to Videogame Criticism, Cambridge, MA, The MIT Press.

Bornet, Cyril, Roulet, Daniel de et Kaplan, Frédéric, 2014, « La simulation humaine : le romanfleuve comme terrain d'expérimentation narrative ", Cahiers de Narratologie. Analyse et théorie narratives, $\mathrm{n}^{\circ} 27$, [En ligne], http://journals.openedition.org/narratologie/7042, consulté le 14 juin 2018.

DOI : $10.4000 /$ narratologie.7042. 
Bourassa, Renée, 2010, Les Fictions hypermédiatiques. Mondes fictionnels et espaces ludiques, Montréal, Le Quartanier.

Braffort, Paul, 2002, «L'ordre dans le crime : une expérience cybernétique avec Italo Calvino », [En ligne], http://www.paulbraffort.net/litterature/critique/calvino_crime.html\#3, consulté le 14 juin 2018.

Caillois, Roger, 1967, Les Jeux et les Hommes : le masque et le vertige, Paris, Gallimard, coll. « Folio ». Caïra, Olivier, 2007, Jeux de rôle. Les forges de la fiction, Paris, CNRS Éditions.

Calvino, Italo, 1980, « Cibernetica e fantasmi (appunti sulla narrativa come processo combinatorio) », dans Una pietra sopra, Turin, Einaudi, p. 164-180.

Calvino, Italo, 2003, « Leçons américaines », dans Défis aux labyrinthes : textes et lectures critiques, traduit par Yves Hersant, vol. 2, Paris, Seuil.

Clément, Jean, 1994a, « afternoon, a story : du narratif au poétique dans l'œuvre hypertextuelle », dans Actes du colloque Nord Poésie et Ordinateur, Roubais, Circav-Gerico, [En ligne], http:// hypermedia.univ-paris8.fr/jean/articles/Afternoon.htm, consulté le 14 juin 2018.

Clément, Jean, 1994b, « Fiction interactive et modernité », Littérature, n 96, p. 19-36, [En ligne], http://www.persee.fr/doc/litt_0047-4800_1994_num_96_4_2350, consulté le 14 juin 2018.

Debeaux, Gaëlle, 2017, Multiplication des récits et stéréométrie littéraire : d'Italo Calvino aux épifictions contemporaines, thèse de doctorat, Rennes, Université Rennes 2, [En ligne], https://tel.archivesouvertes.fr/tel-01677450, consulté le 14 juin 2018.

Deleuze, Gilles et Guattari, Félix, 1980, Capitalisme et schizophrénie 2. Mille plateaux, Paris, Minuit.

Fayol, Michel, 1985, Le Récit et sa construction : une approche de psychologie cognitive, Paris, Delachaux et Niestlé.

Genette, Gérard, 1982, Palimpsestes. La littérature au second degré, Paris, Seuil.

Higuinen, Erwan, 2013, « Marguerite Duras au coeur d'un jeu vidéo », Les Inrockuptibles, 27 janvier 2013, [En ligne], https://www.lesinrocks.com/2013/01/27/medias/marguerite-duras-au-coeurdun-jeu-video-11345549/, consulté le 14 juin 2018.

Hogan, Patrick Colm, 2003, Cognitive Science, Literature and the Arts, New York, Londres, Routledge.

Huizinga, Johan, 1951, Homo ludens : Essai sur la fonction sociale du jeu, trad. par Cécile Seresia, Paris, Gallimard, coll. « Tel ».

Jenkins, Henry, 2004, « Game Design as Narrative Architecture », dans N. Wardrip-Fruin et P. Harrigan (dir.), First Person : New Media as Story, Performance, and Game, Cambridge, MIT Press, p. $117-130$.

Kee, Kevin et Rockwell, Geoffrey, 2011, « The Leisure of Serious Games : A Dialogue », Game Studies, vol. 11, n 2, [En ligne], http://gamestudies.org/1102/articles/

geoffrey_rockwell_kevin_kee, consulté le 14 juin 2018.

Lescure, Jean, 1973, « Petite histoire de l'Oulipo », dans Oulipo, La littérature potentielle (Créations, re-créations, récréations), Paris, Gallimard.

Méchoulan, Éric, 2003, « Intermédialités : le temps des illusions perdues », Intermédialités, $\mathrm{n}^{\circ} 1$, p. 9-27, [En ligne], http://id.erudit.org/iderudit/1005442ar, consulté le 14 juin 2018.

DOI : 10.7202/1005442ar. 
Murray, Janet Horowitz, [1997] 2000, Hamlet on the Holodeck. The Future of Narrative in Cyberspace, Cambridge, MA, The MIT Press.

Picard, Michel, 1986, La Lecture comme jeu, Paris, Minuit.

Queneau, Raymond, 1973b, « Qu'est-ce que l'art? », dans Le voyage en Grèce, Paris, Gallimard.

Rothstein, Edward, 1994, "A New Art Form May Arise From the Myst », The New York Times, 4 décembre 1994, [En ligne], http://www.nytimes.com/1994/12/04/arts/a-new-art-form-mayarise-from-the-myst.html, consulté le 14 juin 2018.

Roubaud, Jacques, 2008, « ALAMO : Rimbaudelaires », 2 avril 2008, [En ligne], http:// www.alamo.free.fr/programmes/rimbaudelaires.html, consulté le 14 juin 2018.

Ryan, Marie-Laure, 2015, Narrative as Virtual Reality 2 : Revisiting Immersion and Interactivity in Literature and Electronic Arts, Baltimore, John Hopkins University Press.

Venerandi, Fabrizio, 2017, Poesie elettroniche, Gênes, Quintadicopertina.

Wittgenstein, Ludwig, [1953] 2004, Recherches philosophiques, trad. par Françoise Dastur, Maurice Élie, Jean-Luc Gautero, Dominique Janicaud et Élisabeth Rigal, Paris, Gallimard.

Zabban, Vinciane, 2012, « Retour sur les game studies. Comprendre et dépasser les approches formelles et culturelles du jeu vidéo », Réseaux, n 173-174 (3), p. 137-76, [En ligne], https:// www.cairn.info/revue-reseaux-2012-3-page-137.htm, consulté le 14 juin 2018.

Zimmerman, Eric, 2004, « Narrative, Interactivity, Play, and Games: Four Naughty Concepts in Need for Discipline », dans P. Harrigan et N. Wardrip-Fruin (dir.), First Person : New Media as Story, Performance, and Game, Cambridge, MA, MIT Press, p. 154-164.

\section{Corpus}

\section{Jeux vidéo cités}

Crowther, William, 1976, Colossal Cave Adventure. PC.

Cyan Worlds, 1993, Myst. PC. Brøderbund Software.

Id Software, 1993, Doom, PC, Id Software.

Quinn, Zoe, 2013, Depression Quest, PC, The Quinnspiracy, http://www.depressionquest.com/\#topsection.

Tale of Tales, 2013, Bientôt l'été, PC, Tale of Tales.

The Astronauts, 2014, The Vanishing of Ethan Carter, PC, Nordic Games.

The Fullbright Company, 2013, Gone Home, PC, The Fullbright Company.

\section{Euvres littéraires citées}

Calvino, Italo, [1973a] 2012, Il castello dei destini incrociati, Milan, Mondadori.

Calvino, Italo, [1973b] 1995, « L'incendio della casa abominevole », dans Romanzi e racconti, i

Meridiani, Milan, Mondadori, vol. 3, p. 319-332.

Calvino, Italo, 1979, Se una notte d'inverno un viaggiatore, Turin, Einaudi.

Cortázar, Julio, 1967, Marelle, trad. par Laure Guille et Françoise Rosset, Paris, Gallimard. 
Joyce, Michael, 1987, afternoon, a story, Eastgate Systems.

Perec, Georges, 1978, La Vie mode d'emploi, Paris, Hachette.

Perec, Georges, [1973] 2008, L'art et la manière d'aborder son chef de service pour lui demander une augmentation, Paris, Hachette.

Queneau, Raymond, 1961, Cent mille Milliards de poèmes, Paris, Gallimard.

Queneau, Raymond, [1967] 1981, « Un conte à votre façon », dans Contes et propos, Paris, Gallimard.

Queneau, Raymond, 1973a, Exercices de style, Paris, Gallimard.

\section{NOTES}

1. En effet, des œuvres comme la Divine Comédie de Dante ou le Décaméron de Boccace fonctionnent comme des récits-cadre enfermant à leur tour différents niveaux narratifs.

2. Cette ambition part aussi d'une opposition à la méthode surréaliste que Queneau n'hésite pas en 1938 à comparer à la méthode de l'entomologue qui se contente de regarder les insectes : le surréaliste « attend bouche bée l'inspiration comme l'entomologue l'insecte qu'il veut capturer ou comme le météorologue la pluie et les météores; à la fin de l'année le météorologue fait un almanach et le poète un recueil de poèmes " (Queneau 1973b : 92).

3. Aujourd'hui, l'intelligence artificielle, assistée par un humain, est capable de composer un roman si on lui donne un nombre suffisant d'informations, comme en témoigne le prix Hoshi Shinichi au Japon qui récompense les œuvres co-écrites par des ordinateurs. Un autre projet, en peinture cette fois-ci, se rapproche davantage des hypothèses de Calvino : il s'agit de " The Next Rembrandt ", une peinture inédite de Rembrandt, et pour cause : il a été créé intégralement par une machine qui a analysé le reste de la production du peintre pour imiter son style (https:// www.nextrembrandt.com/, consulté le 14 juin 2018).

4. Cela étant, il ne fallait pas attendre l'informatique pour imaginer une narration combinatoire. Dans Le château des destins croisés (Calvino 1973a), l'auteur propose des récits de différents personnages s'imbriquant les uns dans les autres au moyen d'un jeu de cartes. Là aussi, le nombre de combinaisons est limité, sans que le chiffre soit précisé, et le récit n'est qu'une actualisation des nombreuses possibilités d'histoires laissées par le jeu de tarot.

5. Le terme est d'autant plus adapté qu'il correspond au "browsing» anglais, lequel sert à désigner la navigation sur Internet.

6. L'intermédialité, introduite par Éric Méchoulan (2003), prend acte de la multiplication des œuvres ayant recours à différents langages médiatiques (texte, image, son, etc.). Ce faisant, c'est le rapport entre ces différents langages qui contribue à produire une signification particulière, qu'il s'agisse d'un rapport de complémentarité ou de "friction ». L'approche intermédiale ne se limite pas aux œuvres contemporaines, mais peut aussi s'appliquer aux œuvres qui n'ont pas été conçues dans cette optique.

7. On retrouve ici la distinction opérée par Gilles Deleuze et Félix Guattari dans Mille Plateaux entre l'arbre et le rhizome : «Un rhizome ne commence et n'aboutit pas, il est toujours au milieu, entre les choses inter-être, intermezzo. L'arbre est filiation, mais le rhizome est alliance, uniquement d'alliance » $(1980: 36)$.

8. Par exemple, ce recueil intitulé Poesie elettroniche (Venerandi 2017) qui multiplie les jeux formels, en présentant une "poésie qui n'est pas sûre de ce qu'elle veut dire " (avec certains mots qui changent sous le regard du lecteur), ou bien une « poésie qui se lit de nuit» (en fonction de l'heure à laquelle on la regarde, elle sera plus ou moins longue). 
9. À l'occasion d'une journée d'études intitulée « Hypertexte et hypertextualité : entre humanités numériques et jeux vidéo », organisée le 12 février 2018 par Daniela Vitagliano et Martin Ringot à l'université d'Aix Marseille.

10. On lira à ce sujet l'article de Vinciane Zabban (2012) qui étudie ces deux tendances et montre pourquoi il faut les dépasser.

11. Olivier Caïra, dans Jeux de rôle. Les forges de la fiction (2007), voit dans le jeu de rôle papier (dont le principal représentant est Dungeons \& Dragons) la forme la plus aboutie et la plus complète de création narrative interactive: "Jamais on a poussé aussi loin l'interactivité qu'avec cette pratique "papier-crayon", quoi qu'en dise l'industrie du jeu vidéo » (2007 : 9). En effet, précise-til, « un scénario de jeu de rôle n'est ni linéaire, à la manière d'un roman, ni arborescent, à la manière des livres interactifs ou des "jeux de rôle" informatiques" (2007:56); c'est plutôt une masse d'informations (univers, personnages, quête) mise à disposition du maître du jeu, un récit potentiel qui ne sera actualisé que lors de la partie : «le scénario d'un jeu de rôle s'adresse au MJ [Maître du Jeu] et non aux joueurs; il procède de la quête et non du récit. La différence ? Une quête s'inscrit dans l'ordre de l'action ; un récit dans l'ordre de la contemplation » (2007: 59).

12. Programme développé dès 1966 par Joseph Weisenbaum, capable de soutenir une conversation avec un utilisateur. ELIZA marque un tournant dans la conception des environnements numériques selon Janet Murray ([1997] $2000: 68$ ).

13. C'est notamment grâce au travail des équipes de Lucasfilm Games, plus tard renommé LucasArts, que ce changement a pu avoir lieu. L'équipe a développé des outils facilitant grandement la conception de ce genre de jeu, notamment «SCUMM » (Script Creation Utility for Maniac Mansion »).

14. Comme le montre l'article dans le New York Times, un an après la sortie du jeu, d'Edward Rothstein qui, faisant un panorama de la scène vidéoludique, voit dans Myst la promesse d'un tournant artistique du jeu vidéo (Rothstein 1994).

15. On pense notamment à la sortie, la même année, de Doom (Id Software 1993), jeu de tir à la première personne et autre titre ayant installé un paradigme ludique, celui du First-PersonShooter (FPS).

16. Il est intéressant d'effectuer ce saut dans le temps pour voir comment les intentions premières des jeux des années 1980 et 1990 se sont traduites à une époque où les outils de développement, plus faciles d'accès, ont permis une profusion de créations vidéoludiques.

17. On reprend ces notions à Roger Caillois dans son essai Les jeux et les Hommes: le masque et le vertige (1967), connu, avec Homo ludens de Johan Huizinga (1951), pour avoir donné naissance à l'étude du jeu, et pour avoir fondé les catégories, toujours d'actualité, de ludus (le jeu réglé, structuré), paidia (le jeu comme pulsion primaire), ainsi que les différentes caractéristiques des différents jeux que sont l'agôn (compétition), la mimicry (le simulacre), l'alea (la chance) et l'ilinx (le vertige).

18. Nous avons décidé pour traduire ces propositions d'employer l'infinitif pour un temps qui dans le cas présent en anglais peut indiquer tant la deuxième personne (comme c'est censé être le cas, étant donné que les propositions suivent le début de question « Do you... » - «Est-ce que vous... ») que la forme employée dans les commandes sur l'ordinateur, rendue en français par l'infinitif. Par ailleurs, le début de question en anglais ne peut fonctionner pour toutes les propositions, notamment la dernière, dont la première phrase finit par un point. Cependant, notre solution nous oblige à effacer les marques de deuxième personne présentes dans le texte.

19. Nom donné à un scandale lié à une possible collusion entre les médias spécialisés et les éditeurs de jeux vidéo, qui a surtout été le lieu d'attaques misogynes et sexistes envers certaines développeuses et journalistes parmi lesquelles, entre autres, Zoe Quinn et Anita Sarkeesian, chercheuse travaillant notamment sur le traitement réservé aux personnages féminins dans les jeux vidéo. On lira à ce propos la synthèse de William Andureau parue dans la rubrique «Pixels » du Monde le 15 septembre 2014 (Audureau 2014). 
20. En effet, dans «The Leisure of Serious Games: A Dialogue » (2011), Geoffrey Rockwell et Kevin Kee jouent un dialogue reprenant les codes de l'échange socratique à l'occasion de la conférence à propos de l'interaction dans les mondes immersifs en 2009. La question initiale est de savoir si un jeu peut être sérieux, et aboutit à un débat sur la frontière entre le jeu et l'apprentissage.

21. Organisé à Toulouse et Montpellier par Claire Chatelet, Amanda Rueda et Julie Savelli en avril 2016 ; publication des actes en cours.

22. Nous laisserons de côté ce niveau qui gagnerait à être étudié dans un article à part.

\section{RÉSUMÉS}

Notre but dans cet article est de montrer en quoi une convergence entre littérature et jeux vidéo peut être trouvée dans l'emploi par les deux médias de la narration interactive. L'évolution de la littérature moderne dans la seconde moitié $d u x^{e}$ siècle l'a amenée au recours à des structures hypertextuelles. Au même moment, les jeux vidéo ont intégré la construction narrative qui a rendu la frontière entre ce nouveau médium et la littérature expérimentale plus poreuse. Nous entendons donc dans cet article montrer que la distinction entre littérature et jeu vidéo n'est pas aussi nette qu'il n'y paraît, et proposer l'établissement d'un spectre reposant sur les niveaux d'interactivité énoncés par Eric Zimmerman.

My goal in this article is to show how a convergence between literature and video games can be found in the use of both medias of interactive narrative. The evolution of modern literature in the late twentieth century led to the resort of hypertextual structures. In the meantime, video games integrated narrative construction which blurred the lines between this new media and experimental literature. Therefore, the present article shows that the distinction between literature and videogames is not as sharp as we may think, to then suggest a configuration of a spectrum based on the interactivity levels stated by Eric Zimmerman.

\section{INDEX}

Keywords : Oulipo, text, hypertext, writing process, Depression Quest, video games, interactive narrative

Mots-clés : Oulipo, texte, hypertexte, jeux vidéo, pratiques d'écriture, Depression Quest, narration interactive

\section{AUTEUR}

\section{MARTIN RINGOT}

Aix Marseille Univ, CAER, Aix-en-Provence, France 